\title{
Comparison of antioxidant capacities of different types of tea using the spectroscopy methods and semi-empirical mathematical model
}

\author{
Mariola Bartoszek $^{1} \cdot$ Justyna Polak $^{1} \cdot$ Mirosław Chorążewski $^{1}$
}

Received: 30 June 2017 / Revised: 5 September 2017 / Accepted: 15 September 2017 / Published online: 30 September 2017

(C) The Author(s) 2017. This article is an open access publication

\begin{abstract}
The antioxidant properties of different types of tea-green, black and earl grey-were investigated using the EPR spectroscopy method based on a new semi-empirical mathematical model and on a single EPR spectroscopy measurement. The obtained values of the antioxidant capacity were correlated with the bioactive ingredient content identified through the NMR spectroscopy. Moreover, an attempt was made to determine what impact on the antioxidant properties of tea does adding sugar, milk, lemon and honey have. All studied teas exhibited antioxidant properties. The best DPPH free radical scavengers were green teas. Adding sugar, milk and lemon juice to tea did not significantly impact the antioxidant properties of the infusion; adding honey, however, caused an increase in the total antioxidant capacity of the infusion. The aromatic proton content correlates positively with the antioxidant capacity value.
\end{abstract}

Keywords Antioxidant capacity $\cdot$ EPR spectroscopy · NMR spectroscopy $\cdot$ DPPH $\cdot$ Tea

\section{Introduction}

In recent years, there has been a growing interest in the antioxidant properties of food products [1-6]. Various methods are used to study the antioxidant capacity of food products,

Electronic supplementary material The online version of this article (doi:10.1007/s00217-017-2986-z) contains supplementary material, which is available to authorized users.

Mariola Bartoszek

mariola.bartoszek@us.edu.pl

1 Institute of Chemistry, The University of Silesia, 9 Szkolna Street, 40-006 Katowice, Poland including tea; these methods are based on different mechanisms, using a variety of detection techniques and are therefore not standardized. Hence, the comparison of the antioxidant capacity values obtained in different laboratories is not possible. On the other hand, the antioxidant capacity values obtained in the same laboratory through the use of different methods and parameters are also not consistent, due to the use of different interactions. Therefore the research is leading to the modification of existing methods and new methods are constantly sought. Recently, an EPR method based on a new semi-empirical mathematical model were proposed [5]. This method allows for a quick and precise determination of the antioxidant capacity of food products. In this paper this method was applied to tea. Tea is one of the most widely consumed beverages in the world [7]. It is valued both for the taste and for the health benefits associated with its antioxidant content [1,7-10]. All varieties of tea are produced from young, tender leaves of Camellia sinensis ( $L$.) (family Theaceae). Through using a wide variety of methods, different types of tea are produced: unfermented (green and white), partially fermented (oolong) and fermented (black and red). Green tea is produced through treating the leaves with hot steam and subsequently drying them. In such conditions, polyphenol oxidation is inactivated, which prevents the oxidation of polyphenols. As a result, the raw material remains rich in flavonols such as: catechin, epicatechin, epigallocatechin, flavonoids and simple phenolic acids [7, 11]. Fermentation is necessary in order to produce black tea. This process consists of enzymatic oxidation by endogenous polyphenol oxidases and peroxidases of tea polyphenols, namely colorless flavonols that are partially converted into theaflavins and thearubigins, responsible for the characteristic aroma and color of the black and oolong teas. About $75 \%$ of the catechin contained in leaves undergoes enzymatic oxidation [12]. 
The basic bioactive ingredients in tea are polyphenols. Both the quantity and the composition of polyphenol compounds in tea varies, thus individual teas are characterized by a different antioxidant potential [7, 13-15].

The major polyphenol components in green tea are the flavanols: catechins, (+)-catechin, (-)-epicatechin (EC), (-)-epicatechin-3-gallate (ECG), (-)-epigallocatechin (EGC), and (-)-epigallocatechin-3-gallate (EGCG), which are known to contribute to many biological functions, including antioxidant activities [16, 17]. The pharmacological significance of catechins decreases sequentially: EGCG $>$ EGC $>$ ECG $>$ EC. In fact, tea catechins are the most powerful antioxidants among known plant phenols. In some laboratory tests, EGCG is 20 times more active than vitamin C, and 30 times more than vitamin E [18]. Tea also contains flavonols, mainly quercetin, kaempferol, myricetin, and their glycosides. In black tea, the oxidation of polyphenols during processing leads to formation of theaflavins, theaflavinic acids, thearubigins or theasinensis, and proanthocyanidin polymers [19]. Tea also contains vitamins E, K, $\mathrm{A}$, and, in the case of green tea, low levels of B vitamins and vitamin $\mathrm{C}[18,20]$. Black tea is also a source of monomer gallate, phenolic acid, caffeine, theobromine and theophylline, and its aroma is enriched by linalool. Additionally, tea contains theanine, which is currently being intensively studied in terms of biological activity [21].

The immense variety of teas available incites interest in the potential advantages presented by each of them for the human body. To date, many studies comparing different varieties of tea have been conducted [1, 7, 12, 16, 22]. Most of these studies demonstrate that antioxidant activity decreases in the following order: green tea $>$ oolong $>$ black tea $[12,22]$, whereas some authors observe that black tea has higher activity than green, and others do not observe significant differences in their antioxidant properties [11]. The preparation method also influences the antioxidant properties of tea. Studies on the impact of tea brewing techniques on flavonoid, catechin, thearubigin and theaflavin content in the black tea extracts have demonstrated that with increased brewing time the content of polyphenol compounds increased as well [10]. The temperature at which tea is brewed also influences its antioxidant properties. Additionally, a common practice is to add enhancements to tea, such as milk, sugar, lemon or honey. The results of studies on the effects of such additions on the antioxidant properties of tea are contradictory and therefore require further research [17].

The goal of the present study was to compare the antioxidant capacity of green, black and earl grey tea using an EPR method based on a new semi-empirical mathematical model. This method allows researchers to reduce time and costs associated with determining the TEAC value more than 5 times, compared with the traditional methods [5].
Moreover, an attempt was made to determine the impact of adding sugar, milk, lemon and honey on the antioxidant properties of tea. The obtained TEAC values were correlated with the content of bioactive ingredients identified through the NMR spectroscopy method.

\section{Materials and methods}

Commercially available samples of black, green and earl grey tea have been used in monitoring their antioxidant properties using an EPR spectroscopy method based on a new semi-empirical mathematical model.

\section{Reagents and equipment}

1,1-diphenyl-2-picrylhydrazyl (DPPH) (Sigma-Aldrich, Poznań, Poland) was used as the source of free radicals. Trolox (molecular formula $\mathrm{C}_{14} \mathrm{H}_{18} \mathrm{O}_{4}$ ) (Acros Organics, Geel, Belgium) was used to quantify the antioxidant activity of the teas. All other chemicals and solvents were of analytical grade and used without additional purification.

Electron paramagnetic resonance (EPR) spectra were obtained with a Bruker EMX EPR spectrometer (BrukerBiospin, Germany) operating at the X-band frequency $(9.8 \mathrm{GHz})$ at room temperature. Typical instrument parameters were: central field, $3480 \mathrm{G}$; modulation amplitude, $2.0 \mathrm{G}$; time constant, 40.96; gain, $1 \times 10^{4} \mathrm{G}$; microwave power, $20.12 \mathrm{~mW}$; sweep width, $60 \mathrm{G}$.

\section{Samples}

Eighteen samples of tea, produced by six manufacturers (P1-P6) were purchased in local stores. Black tea (B1-B6), green tea (G1-G6) and earl grey tea (E1-E6) from each manufacturer were tested. Moreover, for the selected black tea (B1), antioxidant capacity TEAC of the infusion was measured after the addition of substances such as: sugar, lemon juice, milk and honey, and the antioxidant capacities of just the substances (refined sugar, lemon juice, milk and wildflower honey) were determined.

An infusion was prepared from each tea by pouring $100 \mathrm{~mL}$ of boiling water over one tea bag. Due to the fact that the studied teas were sold in tea bags with different weights, the results were recalculated per $1 \mathrm{~g}$ of tea.

Fresh lemon juice was obtained from lemon by squeezing, and measured immediately after preparation. The impact of adding lemon juice on the antioxidant properties of the infusion was tested by adding $20 \mathrm{~mL}$ of lemon juice to $100 \mathrm{~mL}$ of the examined tea infusion. Sugar, purchased in a local store, was added to the tea in the amount of $20 \mathrm{~g}$ per $100 \mathrm{~mL}$ of the tea infusion undergoing examination. Milk with the $3.2 \%$ fat content was also added to the tea in amount usually 
used by the consumers ( $20 \mathrm{~mL} / 200 \mathrm{~mL}$ of infusion). The tea with wildflower honey was tested after adding $30 \mathrm{~g}$ of honey per $100 \mathrm{~mL}$ of infusion. The honey itself was also tested through dissolving $3 \mathrm{~g}$ of honey in $5 \mathrm{~mL}$ of water. Given the varied amounts of tea in the tea bags, the values were also converted to $\mu$ mol trolox per $1 \mathrm{~g}$ of tea.

\section{Determination of antioxidant capacity using EPR method based on a new semi-empirical mathematical model}

Trolox equivalent antioxidant capacity (TEAC) was determined using an EPR method based on a new semi-empirical mathematical model described previously [5]. The mathematical model was based on three variables: $x=V, y=\% I$, and $z=I_{100}$, where $V$-is the volume of antioxidant added to the DPPH free-radical solution $[\mathrm{mL}], \% \mathrm{I}$-is the inhibition [\%] of the EPR signal of the DPPH free-radical, $I_{100}$-is the value calculated from the Eq. (1) describing the relationship between $\% I$ and $V$ corresponding to a $100 \mathrm{~mL}$ sample.

$z(x, y)=a_{o}+a_{1} y+a_{2} y^{2}+a_{3} y^{3}+\frac{a_{4}+a_{5} y+a_{6} y^{2}}{x}+\frac{a_{7}+a_{8} y}{x^{2}}+\frac{a_{9}}{x^{3}}$

where $a_{0}=f_{0} g_{0}, a_{1}=f_{0} g_{1}, a_{2}=f_{0} g_{2}, a_{3}=f_{0} g_{3}, a_{4}=f_{1} g_{0}$, $a_{5}=f_{1} g_{1}, a_{6}=f_{1} g_{2}, a_{7}=f_{2} g_{0}, a_{8}=f_{2} g_{1}, a_{9}=f_{3} g_{0}$ (Table 1).

The inhibition percentage of the EPR spectrum $\% I$ was calculated according to the following equation: $\% I=\left[\left(I_{0}-I\right) / I_{0}\right] \times 100 \%$, where $I_{0}$ is the area of the EPR spectrum of DPPH (control sample), and $I$ is the area of the EPR spectrum of sample DPPH. The surface area was calculated by double integral of the entire obtained DPPH spectrum (Fig. 1).

Three infusions were prepared for each tested tea. For two selected volumes of antioxidant sample added to $1 \mathrm{~mL}$ of $200 \mu \mathrm{mol} / \mathrm{dm}^{3} \mathrm{DPPH}$ solution in ethanol, the EPR spectrum was measured and $\% I$ of the DPPH free-radical of EPR signal was determined. Based on the $V$ value of the antioxidant added to the free-radical solution, as well as $\% I$ of the

Table 1 Coefficients of Eq. (1) together with the adjusted coefficient of determination for the nonlinear regression, adj- $R^{2}$

\begin{tabular}{ll}
\hline$a_{0}$ & $-50924 \pm 1005$ \\
$a_{1}$ & $-1493 \pm 110$ \\
$a_{2}$ & $47.23 \pm 7.63$ \\
$a_{3}$ & $-0.277 \pm 0.012$ \\
$a_{4}$ & $2842 \pm 440$ \\
$a_{5}$ & $32.887 \pm 8.399$ \\
$a_{6}$ & $0.446 \pm 0.058$ \\
$a_{7}$ & $-4.782 \pm 0.39$ \\
$a_{8}$ & $-0.0438 \pm 0.0095$ \\
$a_{9}$ & $0.00329 \pm 0.00047$ \\
adj- $R^{2}$ & 0.91 \\
\hline
\end{tabular}

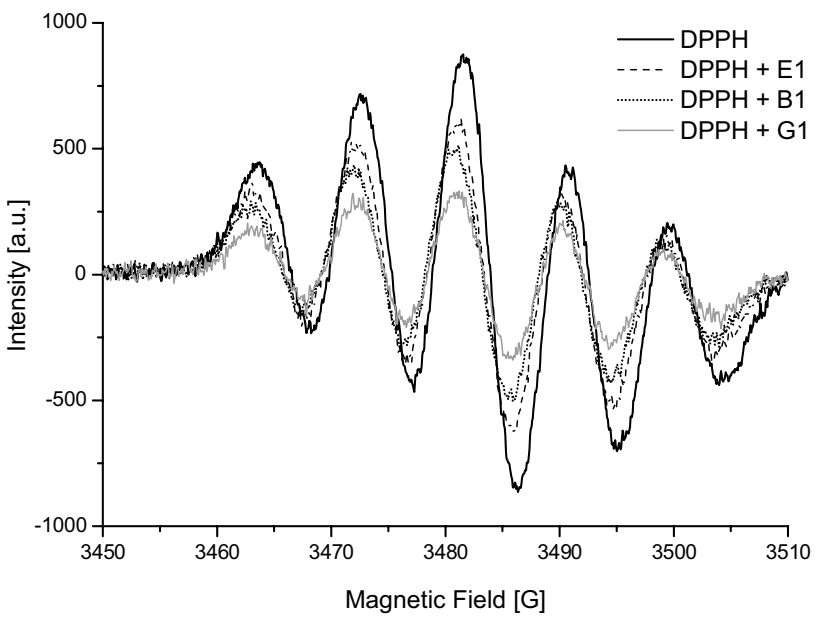

Fig. 1 EPR spectra of DPPH and DPPH with black (B1), green (G1) and earl grey (E1) teas

DPPH free-radical EPR signal, the $I_{100}$ value was determined using Eq. (1) (raw data in the Supplemental Materials is presented). Next, based on the $I_{100}$ value the antioxidant capacity TEAC, given in $\mu \mathrm{mol}$ trolox per $100 \mathrm{~mL}$ of sample or per $1 \mathrm{~g}$ of sample, was calculated according to the regression equation $I_{100}=987.60 \mathrm{TEAC}+16.36$. The data presented in Table 2 are the result of three trials.

\section{NMR spectroscopy}

${ }^{1} \mathrm{H}$ NMR spectra were recorded using the Bruker Ascend NMR spectrometer, operating at $500 \mathrm{MHz}$ for ${ }^{1} \mathrm{H}$ nuclei with a $5 \mathrm{~mm}$ indirect detection pulsed field gradient probe. The temperature during all experiments was controlled at $298 \mathrm{~K}$. For water suppression, the pre-saturation method was used. The samples for NMR analysis were prepared as follows: $0.3 \mathrm{~mL}$ of tea was transferred into a $5 \mathrm{~mm}$ NMR tube, and mixed with $0.3 \mathrm{~mL} \mathrm{D}_{2} \mathrm{O} .64$ scans of $32 \mathrm{~K}$ data points were acquired with a spectral width of $16 \mathrm{ppm}$, acquisition time of $5.45 \mathrm{~s}$, and relaxation delay of $5 \mathrm{~s}$. Spectrum processing was performed using TOPSPIN 3.1 Bruker software. The FIDs were Fourier transformed, the spectra were phased, the baseline corrected and the relative integrals of the aromatic region (6.2-8.2 ppm) were evaluated [6].

\section{Results and discussion}

\section{Comparison of the antioxidant capacities of teas}

The results of our studies show that EPR method based on a new semi-empirical mathematical model is an adequate to analyze the antioxidant activity of food products. 


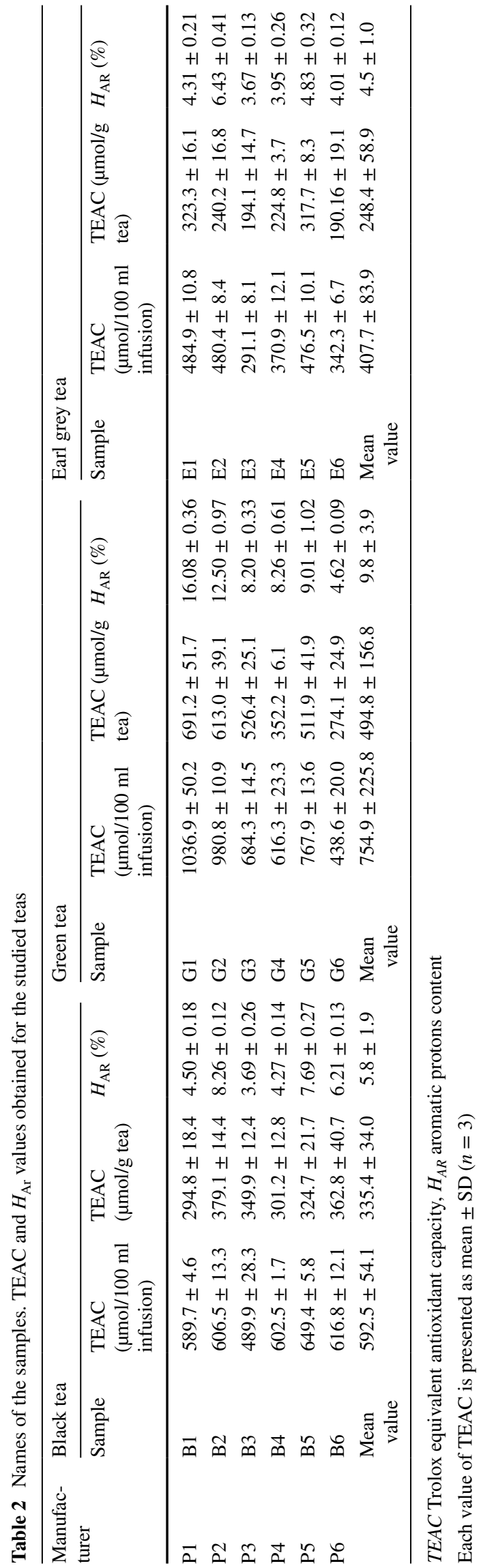

Based on the mathematical model, the total antioxidant capacity TEAC was determined (Table 2) for each of the studied teas.

No total antioxidant capacity TEAC values determined by EPR spectroscopy were found in the literature. However, TEAC values obtained in this work are congruent with the values determined through the use of other research methods [1, 10, 23]. TEAC values found in literature varied between 143 and 3100 ( $\mu$ mol Trolox/100 mL) for black tea $[1,10,16,21,24]$, between 480 and 6200 $(\mu \mathrm{mol} \mathrm{TE} / 100 \mathrm{~mL})$ for green tea $[16,17,21]$ and between 728 and 1205 ( $\mu \mathrm{mol}$ Trolox/100 mL) for earl grey tea [17]. All TEAC values obtained in the present study, for all tea types, are located within the lower limits of values given in the literature. Results of the total antioxidant capacity TEAC values obtained for black, green and earl grey teas from individual manufacturers were analyzed (Table 2). This analysis allowed to determine differences in the antioxidant properties of teas depending on their production method. Green tea was the best DPPH free radical scavenger, with average TEAC value amounting to $495 \pm 157$ $(\mu \mathrm{mol} / 1 \mathrm{~g})$. Black tea was characterized by lower antioxidant activity, with average TEAC value equaling $335 \pm 34$ $(\mu \mathrm{mol} / 1 \mathrm{~g})$, and the lowest was exhibited by earl grey tea, with an average TEAC value of $248 \pm 59(\mu \mathrm{mol} / 1 \mathrm{~g})$ (Table 2). Only for manufacturer P6, a higher TEAC value was noted for black tea as compared with green and earl grey tea (Table 2).

In general, the obtained results confirm the higher TEAC value of unfermented green tea than of fermented black tea, which is in line with data found in literature [12, $13,25]$, although some studies indicate a reverse trend [15] or lack of differences in the antioxidant properties of teas manufactured via different technological processes $[1,23]$. In case of earl grey tea, earlier studies indicated a smaller polyphenol content compared to green and black tea [23], which can be explained by a lower total antioxidant potential of earl grey tea, despite the bergamot oil content, renowned for its antioxidant properties [26].

Significant variances in the TEAC values obtained in individual groups (green, black and earl grey tea) depending on the manufacturer are also worth mentioning. In case of green tea, this variance amounts up to $417.1(\mu \mathrm{mol} / 1 \mathrm{~g})$. Such large discrepancies in the TEAC values indicate that not only the production method (fermentation or its absence), but also individual technological processes of eliminating the natural moisture from the tea leaves (socalled wilting), twisting the leaves, duration and method of conducting fermentation, duration and temperature of drying, utilized by different manufacturers, as well as the origins of the tea itself, can determine the antioxidant properties of the product which reaches the consumer. 


\section{NMR spectroscopy}

Catechins, which are most abundant in green teas, are believed to present the greatest impact on the antioxidant activity of tea infusions. Along with an increase in the catechin content comes an increase in the antioxidant activity $[16,17]$. The significantly higher content of catechins in green tea, as compared to black tea, was confirmed by NMR studies, which were conducted for each tea studied. Individual signals were assigned based on data from literature $[27,28]$. The differences are apparent especially in the aromatic proton range, where in the ${ }^{1} \mathrm{H}$ NMR spectra obtained for green teas, signals were observed at 6-6.1 ppm, 6.5-6.6 ppm and 6.8-6.9 ppm, confirming the presence of EGC, EGCG, ECG and EC in green tea; whereas in the black tea and earl grey tea spectrum, the signals in this area have significantly lower intensity and can originate from theaflavins (TF), formed from catechins at the fermentation stage during black tea manufacturing (Fig. 2). Moreover, the signal at $7.1 \mathrm{ppm}$, which is evident in black and earl grey
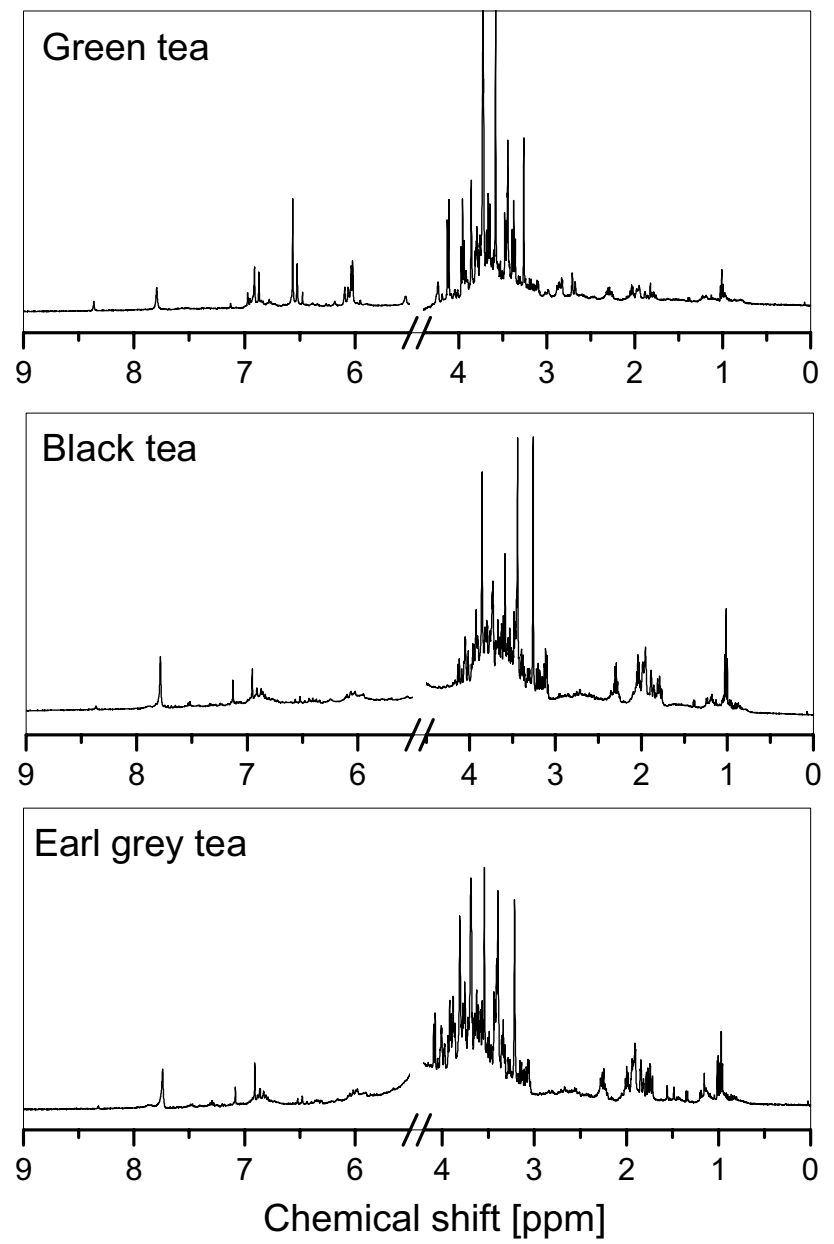

Fig. $2{ }^{1} \mathrm{H}$ NMR spectra of black (B1), green (G5) and earl grey (E5) teas tea's spectra, indicates enriching the tea with gallic acid during the fermentation process.

The obtained NMR spectra thus confirm that the fermentation process changes the antioxidant content of tea both in terms of quantity as well as quality. There are known studies which show that the proton content in aromatic groups correlates with the antioxidant capacity of food products [3, 6]. An analysis of the NMR spectra of the samples tested in this study also confirms this thesis. Green tea, characterized by the highest average TEAC value, also provided the highest $H_{\text {ar }}$ content values (Table 2). The observed decrease in $H_{\text {ar }}$ aromatic protons occurred in the following order: green tea $>$ black tea $>$ earl grey tea. The correlation factor between the aromatic proton content and the antioxidant capacity value is 0.92 for TEAC measured in $(\mu \mathrm{mol} / 100 \mathrm{~mL}$ of infusion) and 0.89 for TEAC measured in $(\mu \mathrm{mol} / 1 \mathrm{~g}$ of tea), which indicates a strong positive correlation between these quantities (Fig. 3).

\section{Influence of additives on the antioxidant properties of tea}

In order to determine the influence on the tea of commonly used additives, antioxidant properties were tested for black tea B1 with addition of: honey, lemon juice, milk, and sugar. The additives themselves-lemon juice and honey-were also tested. These additives were used in quantities that are normally used while drinking tea. Due to the reduction of ascorbic acid in high temperatures, examining the impact of lemon juice on the antioxidant properties of tea was conducted after adding lemon juice to both hot and cold infusions. The TEAC results obtained for the tea with additives were within the range from 515.7 ( $\mu \mathrm{mol} / 100 \mathrm{~mL}$ of infusion)

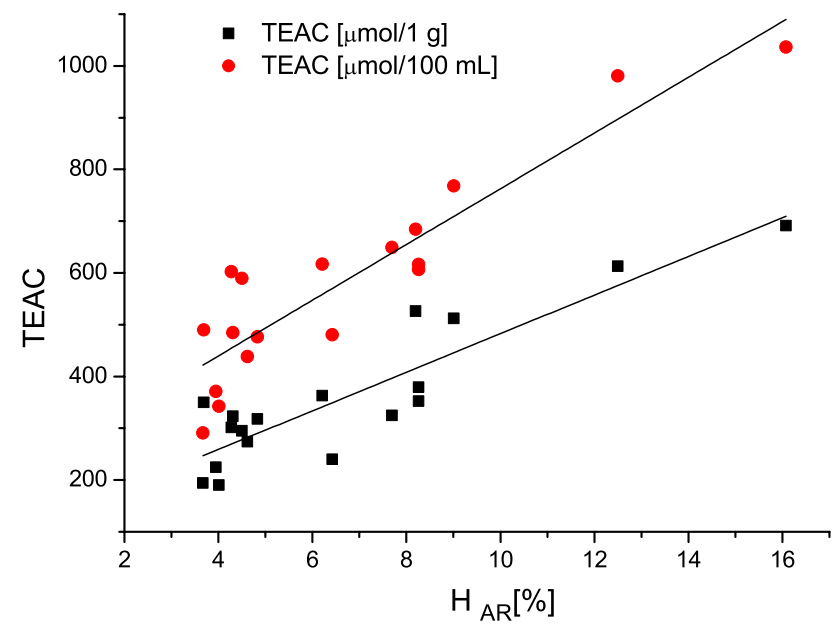

Fig. 3 The correlation between trolox equivalent antioxidant capacity (TEAC) and the aromatic protons $\left(H_{\mathrm{AR}}\right)$ 
for hot tea with added lemon juice, to $668.6(\mu \mathrm{mol} / 100 \mathrm{~mL}$ of infusion) for tea with added honey (Table 3 ).

Among the additives tested, only honey significantly impacted the total antioxidant capacity TEAC values. The other additives contributed to only a slight decrease in the TEAC values, within the margin of error. The lack of influence of sugar on the antioxidant properties of tea is not surprising, as white sugar does not display any antioxidant properties. This is confirmed by both the results of the tests conducted in this study, as well as of studies conducted using the volumetric method [29]. Individual reports show an insignificant effect of sugar on antioxidant values and demonstrate insignificant antioxidant amounts in some varieties of refined and unrefined sugar [29].

Lemon juice, which is known for its antioxidant properties, as well as the ascorbic acid found in lemons, are one of the most popular antioxidants [30]. The decrease in the antioxidant properties of tea after adding lemon juice may therefore seem surprising. The TEAC for lemon juice is $452.3(\mu \mathrm{mol} / 100 \mathrm{~mL})$ (Table 3 ), which explains a slight decrease in the antioxidant properties of tea after adding lemon juice. It thus turned out that a black tea infusion is a better antioxidant than lemon juice. It is worth noticing that the TEAC value for the infusion with added lemon juice was higher after cooling than the TEAC value of the infusion where lemon juice was added while it was still hot. This is most likely caused by the process of thermal degradation of ascorbic acid.

Tea is often consumed with addition of milk, so an analysis of its influence on antioxidant values appears interesting. The addition of milk, similarly to sugar and lemon juice, did not have a significant influence on the antioxidant properties of tea. It has been determined that there are non-covalent interactions between polyphenols contained in tea infusions and polar proteins found in milk. These interactions may influence the antioxidant potential of the polyphenols found in black tea. The results of the research conducted to date

Table 3 The TEAC values obtained for B1 tea with additives and TEAC values for honey and lemon juice

\begin{tabular}{lc}
\hline Sample & TEAC \\
\hline B1 & $589.7 \pm 4.6(\mu \mathrm{mol} / 100 \mathrm{~mL})$ \\
B1 + honey & $668.6 \pm 18.9(\mu \mathrm{mol} / 100 \mathrm{~mL})$ \\
B1 + sugar & $553.0 \pm 20.0(\mu \mathrm{mol} / 100 \mathrm{~mL})$ \\
B1 + milk & $545.6 \pm 17.4(\mu \mathrm{mol} / 100 \mathrm{~mL})$ \\
B1 + lemon juice (hot) & $515.7 \pm 42.4(\mu \mathrm{mol} / 100 \mathrm{~mL})$ \\
B1 + lemon juice (cold) & $571.9 \pm 67.7(\mu \mathrm{mol} / 100 \mathrm{~mL})$ \\
Lemon juice & $452.3 \pm 44.8(\mu \mathrm{mol} / 100 \mathrm{~mL})$ \\
Honey & $0.32 \pm 0.05(\mu \mathrm{mol} / 1 \mathrm{~g})$ \\
\hline
\end{tabular}

TEAC Trolox equivalent antioxidant capacity

Each value of TEAC is presented as mean $\pm \mathrm{SD}(n=3)$ are ambiguous. Some studies indicate a decrease in antioxidant potential of tea after adding milk [31], others signal an increase in the antioxidant value [32]. There are also studies known where no change in the antioxidant properties of tea was observed after adding milk [33].

The only studied additive to significantly impact the antioxidant properties of tea (Table 3) was honey. The antioxidant properties of honey are mainly determined by flavonoids: (chrysin, pinocembrin, pinobanksin, quercetin, kaempferol, luteolin, galangin, apigenin, hesperetin, myricetin), phenolic acids (caffeic, coumaric, ferrulic, ellagic, chlorogenic), ascorbic acid, catalase, peroxidase, carotenoids and products of the Maillard reaction contained in honey $[34,35]$. The amount of these components varies considerably depending on the type of flower and the geographical origin of honey $[34,36]$. Moreover, the processing and storage methods may affect the composition of honey, and subsequently its antioxidant properties [35].

\section{Conclusion}

On the basis of the conducted studies it was concluded that all the examined teas exhibited antioxidant qualities. The best DPPH free radical scavengers were green teas, black teas demonstrated lower antioxidant activity, and the lowest was exhibited by earl grey teas. Significant differences in the total antioxidant potential obtained in individual groups (green, black and earl grey teas) demonstrated that not only the production method (fermentation or its absence), but also individual technological processes utilized by different manufacturers, as well as the origin of the tea itself, can impact the antioxidant properties of the final product which reaches the consumer.

Studies conducted using the NMR spectroscopy method showed the presence of catechins in green tea, as well as a lack of indicators of catechins in black tea. This fact can suggest a conclusion that this compound is mainly responsible for the antioxidant properties of green tea. The aromatic proton content also correlates positively with the TEAC value.

Adding sugar, milk and lemon juice to tea did not have a significant effect on the antioxidant properties of the infusion; however, adding honey caused an increase in the total antioxidant capacity of the infusion.

\section{Compliance with ethical standards}

Confict of interest The authors declare that they have no conficts of interest. 
Compliance with ethics requirements This article does not contain any studies with human or animal subjects.

Open Access This article is distributed under the terms of the Creative Commons Attribution 4.0 International License (http://creativecommons.org/licenses/by/4.0/), which permits unrestricted use, distribution, and reproduction in any medium, provided you give appropriate credit to the original author(s) and the source, provide a link to the Creative Commons license, and indicate if changes were made.

\section{References}

1. Bancirova M (2012) Comparison of the antioxidant capacity and the antimicrobial activity of black and green tea. Food Res Int 43:1379-1382

2. Bartoszek M, Polak J (2012) An electron paramagnetic resonance study of antioxidant properties of alcoholic beverages. Food Chem 132:2089-2093

3. Polak J, Bartoszek M (2015) The study of antioxidant capacity of varieties of nalewka, a traditional Polish fruit liqueur, using EPR, NMR and UV-vis spectroscopy. J Food Compos Anal 40:114-119

4. Polak J, Bartoszek M, Stanimirova I (2013) A study of the antioxidant properties of beers using electron. Food Chem 141:3042-3049

5. Polak J, Bartoszek M, Chorazewski M (2015) Antioxidant capacity: experimental determination by EPR spectroscopy and mathematical modeling. J Agric Food Chem 63:6319-6324

6. Stasko A, Brezova V, Mazur M, Certık M, Kalinak M, Gescheidt $\mathrm{G}$ (2008) A comparative study on the antioxidant properties of Slovakian and Austrian wine. Food Sci Technol Int 41:2126-2135

7. Chen H, Zhang Y, Lu X, Qu Z (2012) Comparative studies on the physicochemical and antioxidant properties of different tea extracts. J Food Sci Tech 49(3):356-361

8. Cooper R, Morre DJ, Morre DM (2005) Medicinal benefits of green tea: part I. Review of noncancer health benefits. J Altern Complem Med http://www.ncbi.nlm.nih.gov/ pubmed/1599223911:521-528

9. Hara Y, Luo SJ, Wickremashinghe RL, Yamanishi T (1995) Uses and benefits of tea. Food Rev Int 11:527-542

10. Venditti E, Bacchetti T, Tiano L, Carloni P, Greci L, Damiani E (2010) Hot vs. cold water steeping of different teas: do they affect antioxidant activity? Food Chem 119:1597-1604

11. El-Moneim A, Afify MR, Shalaby EA, El-Beltagi HS (2011) Antioxidant activity of aqueous extracts of different caffeine products. Not Bot Horti Agrobot 39(2):117-123

12. Khokhar S, Magnusdottir SGM (2002) Total phenol, catechin, and caffeine contents of teas commonly consumed in the United Kingdom. J Agric Food Chem 50:565-570

13. Gadow A, Joubert E, Hansmann CF (1997) Comparison of the antioxidant activity of rooibos tea (Aspalathus linearis) with green, oolong and black tea. Food Chem 60(1):73-77

14. Gursoy N, Sarikurkcu C, Cengiz M, Solak MH (2009) Antioxidant activities, metal contents, total phenolics and flavonoids of seven Morchella species. Food Chem Toxicol 47(9):2381-2388

15. Singleton VL, Orthofer R, Lamuela-Raventos M, Lester P (1999) Analysis of total phenols and other oxidation substrates and antioxidants by means of Folin-Ciocalteu reagent. Method Enzymol 299:152-178

16. Carloni P, Tiano L, Padella L, Bacchetti T, Customu C, Kay A, Damiani E (2013) Antioxidant activity of white, green and black tea obtained from the same tea cultivar. Food Res Int 53:900-908
17. Henning SM, Fajardo-Lira C, Lee HW, Youssefian AA, Go VLW, Heber D (2003) Catechin content of 18 teas and a green tea extract supplement correlates with the antioxidant capacity. Nutr Cancer 45(2):226-235

18. Bancirova M (2013) Black and green tea-how to make a perfect crime. J Forensic Leg Med 20(6):635-639

19. Balentine DA, Wiseman SA, Bouwens LC (1997) The chemistry of tea flavonoids. Crit Rev Food Sci 37:693-704

20. Dufresne C, Farnworth E (2000) Tea, kombucha, and health: a review. Food Res Int 33:409-421

21. Gorjanović S, Komes D, Pastor FT, Belščak-Cvitanović A, Pezo L, Hečimović I, Sužnjević D (2012) Antioxidant capacity of teas and herbal infusions. J Agric Food Chem 60:9573-9580

22. Hilton PJ (1972) In vitro oxidation of flavanols from tea leaf. Phytochemistry 18:1243-1248

23. Lin LY, Juan MI, Chen LY, Liang CY, Lin KJ (1996) Composition of polyphenols in fresh tea leaves and associations of their oxygen-radical-absorbing capacity with antiproliferative actions in fibroblast cells. J Agric Food Chem 44:1387-1394

24. Peterson J, Dwyer J, Jacques P, Rand W, Prior R, Chui K (2004) Tea variety and brewing techniques influence flavonoid content of black tea. J Food Compos Anal 17:397-405

25. Hodgson JM, Proudfoot JM, Croft KD, Puddey I, Mori TA, Beilin LJ (1999) Comparison of the effects of black and green tea on in vivo lipoprotein oxidation in human serum. J Sci Food Agric 79:561-566

26. Bocco A, Cuvelier ME, Richard H, Berset C (1998) Antioxidant activity and phenolic composition of citrus peel and seed extracts. J Agric Food Chem 46:2123-2129

27. Bonnely S, Davis A, Lewis JR, Astill C (2003) A model oxidation system to study oxidised phenolic compounds present in black tea. Food Chem 83:485-492

28. Lee JE, Lee BJ, Chung JO, Shin HJ, Lee SJ, Lee ChH, Hong YS (2011) ${ }^{1} \mathrm{H}$ NMR-based metabolomic characterization during green tea (Camellia sinensis) fermentation. Food Res Int 44:597-604

29. Jocelyn S, Hong-Ben Y, José SH, Khairul-Anwar MA (2010) Cyclic voltammetric analysis of antioxidant activity in cane sugars and palm sugars from Southeast Asia. Food Chem 118:840-846

30. Hyang-Sook C, Sun SH, Hiroyuki U, Masayoshi S (2000) Radical-scavenging activities of citrus essential oils and their components: detection using 1,1-diphenyl-2-picrylhydrazyl. J Agric Food Chem 48(9):4156-4161

31. Vasundhara Sharma H, Vijay Kumar L, Jagan Mohan R (2008) Influence of milk and sugar on antioxidant potential of black tea. Food Res Int 41:124-129

32. Dubeau S, Samson G, Tajmir-Riahi HA (2010) Dual effect of milk on the antioxidant capacity of green, Darjeeling and English breakfast teas. Food Chem 122:539-545

33. Leenen R, Roodenburg AJC, Tijburg LBM, Wiseman SA (2000) A single dose of tea with or without milk increases plasma antioxidant activity in humans. Eur J Clin Nutr 54:87-92

34. Bertoncelj J, Dobersek U, Jamnik M, Golob T (2007) Evaluation of the phenolic content, antioxidant activity and colour of Slovenian honey. Food Chem 105:822-828

35. Lachman J, Orsak M, Hejtmankova A, Kovarova E (2010) Evaluation of antioxidant activity and total phenolics of selected Czech honeys. Food Sci Technol Int 43:52-58

36. Beretta G, Granata P, Ferrero M, Orioli M, Facino RM (2005) Standardization of antioxidant properties of honey by a combination of spectrophotometric/fluorimetric assays and chemometrics. Anal Chem Acta 533:185-191 